\title{
Numerical Integration over the $n$-Dimensional Spherical Shell
}

\author{
By D. Mustard
}

\begin{abstract}
The $n$-dimensional generalisation of a theorem by W. H. Peirce [1] is given, providing a method for constructing product type integration rules of arbitrarily high polynomial precision over a hyperspherical shell region and using a weight function $r^{s}$. Table I lists orthogonal polynomials, coordinates and coefficients for integration points in the angular rules for $3 \mathrm{rd}$ and 7 th degree precision and for $n=3(1) 8$. Table II gives the radial rules for a shell of internal radius $R$ and outer radius 1: (i) a formula for the coordinate and coefficient in the 3rd degree rule for arbitrary $n, R$; (ii) a formula for the coordinates and coefficients for the 7 th degree rule for arbitrary $n$ and $R=0$ and (iii) a table of polynomials, coordinates and coefficients to $9 \mathrm{D}$ for $n=4,5$ and $R=0, \frac{1}{4}, \frac{1}{2}, \frac{3}{4}$.
\end{abstract}

1. Introduction. W. H. Peirce has given a method for constructing product type integration rules of arbitrarily high precision for the circular annulus and the threedimensional spherical shell [1]. This paper generalises his theorem to $n$ dimensions and contains his results as particular cases. The method used is similar to that used by Stroud and Secrest [5] for an infinite solid $n$-sphere and also to that used by Mysovskih [6] for the finite solid $n$-sphere.

Let $x_{1}, x_{2}, \cdots, x_{n}$ be Cartesian coordinates in $n$-dimensional Euclidean space. The region of integration, $D$, is the spherical shell of inner radius $R$, outer radius 1 and with centre at the origin, defined by

$$
R^{2} \leqq \sum_{i=1}^{n} x_{i}^{2} \leqq 1
$$

An integration rule

$$
I \equiv \int_{D} f(\mathbf{x}) d \tau \doteq \sum_{i=1}^{N} \alpha_{i} f\left(\mathbf{x}_{i}\right)
$$

is sought which is of precision $k$, i.e., it is exact for all functions $f\left(x_{1}, x_{2}, \cdots, x_{n}\right)$ that are polynomials in $x_{1}, x_{2}, \cdots, x_{n}$ of at most $k$ th degree and is not exact for some $(k+1)$ st degree polynomial. Transform to $n$-dimensional spherical polar coordinates, $\theta_{1}, \theta_{2}, \cdots, \theta_{n-1}, r$, by the equations

$$
\begin{aligned}
& x_{1}=r \sin \theta_{1} \sin \theta_{2} \cdots \sin \theta_{n-1}, \\
& x_{2}=r \cos \theta_{1} \sin \theta_{2} \cdots \sin \theta_{n-1}, \\
& x_{3}=r \cos \theta_{2} \sin \theta_{3} \cdots \sin \theta_{n-1}, \\
& \text {................. } \\
& x_{n}=r \cos \theta_{n-1} \text {. }
\end{aligned}
$$

Received February 3, 1964. 


\section{TABLE I}

Coordinates and coefficients for the $n$-dimensional sphere angular integration rule $\int_{0}^{\pi} \sin ^{\nu} \phi g(\phi) d \phi \doteq \sum_{i=1}^{2 m+2} b_{i} g\left(\phi_{i}\right), \quad \nu=1,2, \cdots, n-2$,

\begin{tabular}{l|c|c|c}
\multicolumn{2}{c}{$y=\cos \phi$} \\
\hline$\nu$ & Polynomial $Q_{2 m+2}^{(\nu-1) / 2}$ & Coordinates $y_{i}$ & Coefficients $b_{i}$ \\
\hline 1 & $\frac{3}{2}\left(y^{2}-\frac{1}{3}\right)$ & $\pm \frac{1}{\sqrt{3}}$ & 1 \\
2 & $\frac{5}{2}\left(y^{2}-\frac{1}{4}\right)$ & $\frac{\pi}{4}$ \\
3 & $\frac{15}{4}\left(y^{2}-\frac{1}{5}\right)$ & $\pm \frac{\pi}{3}, \cos \frac{2 \pi}{3}$ & $\frac{2}{\sqrt{5}}$ \\
4 & $\frac{21}{4}\left(y^{2}-\frac{1}{6}\right)$ & $\pm \frac{1}{\sqrt{6}}$ & $\frac{3 \pi}{16}$ \\
5 & $7\left(y^{2}-\frac{1}{7}\right)$ & $\pm \frac{1}{\sqrt{7}}$ & $\frac{8}{15}$ \\
6 & $9\left(y^{2}-\frac{1}{8}\right)$ & $\pm \frac{1}{\sqrt{8}}$ & $\frac{5 \pi}{32}$ \\
\hline
\end{tabular}

$m=1$ (7th degree rules)

\begin{tabular}{|c|c|c|c|}
\hline 1 & $\frac{35}{8}\left(y^{4}-\frac{6}{7} y^{2}+\frac{3}{35}\right)$ & $\begin{array}{l} \pm \sqrt{\frac{15+2 \sqrt{30}}{35}} \\
\pm \sqrt{\frac{15-2 \sqrt{30}}{35}}\end{array}$ & $\begin{array}{l}\frac{49}{6(18+\sqrt{30})} \\
\frac{49}{6(18-\sqrt{30})}\end{array}$ \\
\hline 2 & $\frac{63}{8}\left(y^{4}-\frac{3}{4} y^{2}+\frac{1}{16}\right)$ & $\begin{array}{l}\cos \frac{\pi}{5}, \cos \frac{4 \pi}{5} \\
\cos \frac{2 \pi}{5}, \cos \frac{3 \pi}{5}\end{array}$ & $\begin{array}{l}\frac{\pi}{8}\left(1-\frac{1}{\sqrt{5}}\right) \\
\frac{\pi}{8}\left(1+\frac{1}{\sqrt{5}}\right)\end{array}$ \\
\hline 3 & $\frac{105}{8}\left(y^{4}-\frac{2}{3} y^{2}+\frac{1}{21}\right)$ & $\begin{array}{l} \pm \sqrt{\frac{7+\sqrt{28}}{21}} \\
\pm \sqrt{\frac{7-\sqrt{28}}{21}}\end{array}$ & $\begin{array}{l}\frac{6}{5}\left(\frac{1}{5-\sqrt{7}}\right) \\
\frac{6}{5}\left(\frac{1}{5+\sqrt{7}}\right)\end{array}$ \\
\hline
\end{tabular}


TABLE I (Continued)

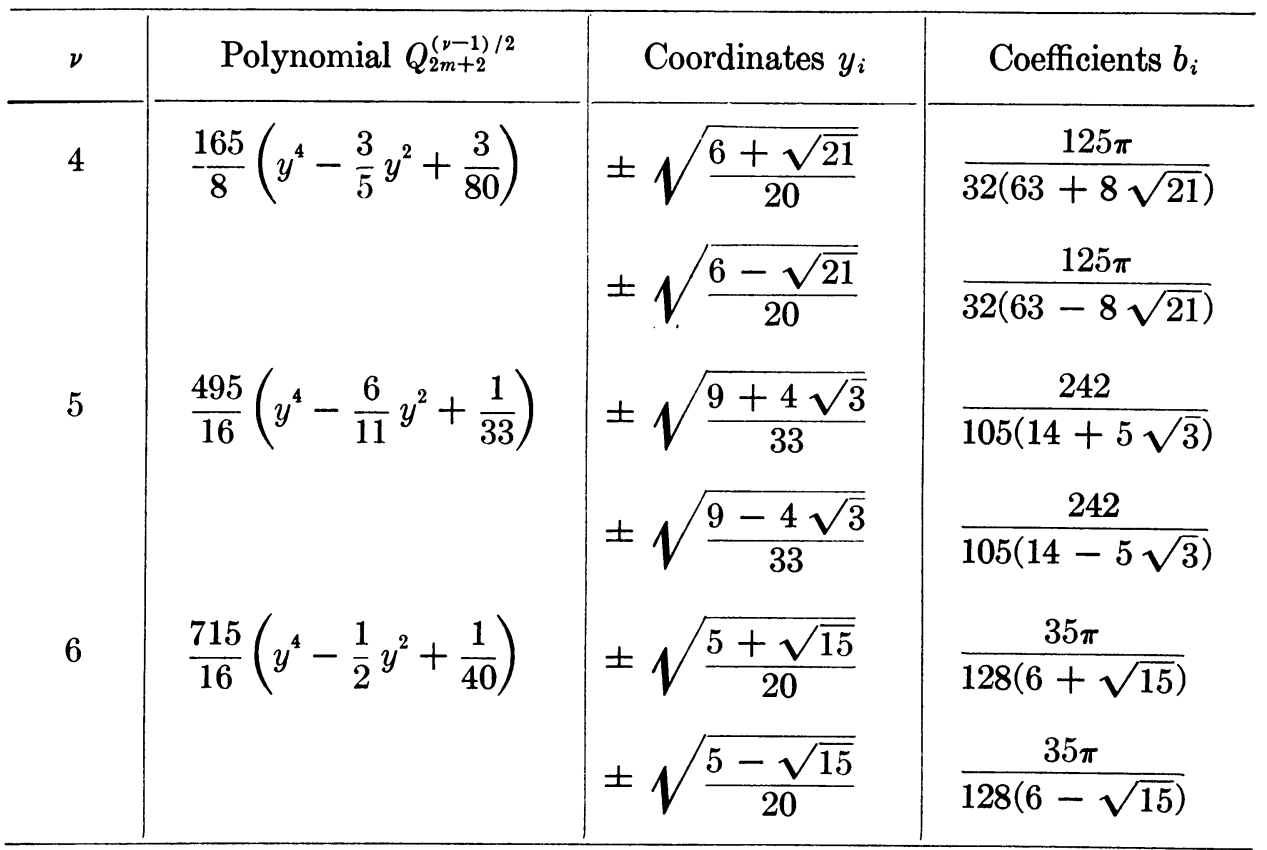

TABLE II

Coordinates and coefficients for the n-dimensional sphere radial integration rule

$$
\begin{gathered}
\frac{\int_{R}^{1} r^{n-1} g\left(r^{2}\right) d r \doteq \sum_{j=1}^{m+1} c_{j} g\left(r_{j}{ }^{2}\right)}{m=0, n, R \text { arbitrary }} \\
\frac{Q_{1}\left(r^{2}\right)=r^{2}-\frac{n}{n+2}\left(\frac{1-R^{n+2}}{1-R^{n}}\right),}{r_{1}{ }^{2}=\frac{n}{n+2}\left(\frac{1-R^{n+2}}{1-R^{n}}\right), \quad c_{1}=\frac{1}{n}\left(1-R^{n}\right)} \\
\frac{m=1, \quad R=0, n \text { arbitrary }}{r_{1}{ }^{2}=\frac{(n+2)(n+4)-2 \sqrt{2(n+2)(n+4)}}{(n+4)(n+6)},} \\
\left.r_{2}{ }^{2}=\frac{(n+2)(n+4)+2 \sqrt{2(n+2)(n+4)}}{(n+4)(n+6)}, \frac{(n-2)(n+4)}{(n+2) \sqrt{2(n+2)(n+4)}}\right\} \\
c_{2}=\frac{1}{2 n}\left\{1+\frac{(n-2)(n+4)}{(n+2) \sqrt{2(n+2)(n+4)}}\right\}
\end{gathered}
$$


NUMERICAL INTEGRATION OVER THE $n$-DIMENSIONAL SPHERICAL SHELL

TABLE II-Continued

$m=1, \quad n=4$ (For $n=3$, see the table of W. H. Peirce.)

\begin{tabular}{|c|c|c|c|}
\hline$R$ & $Q_{2}\left(r^{2}\right)$ & $r_{i}^{2}$ & $c_{i}$ \\
\hline 0 & $r^{4}-\frac{6}{5} r^{2}+\frac{3}{10}$ & $\begin{array}{l}r_{1}^{2}=0.355051024 \\
r_{2}^{2}=0.844948977\end{array}$ & $\begin{array}{l}c_{1}=0.090979310 \\
c_{2}=0.159020691\end{array}$ \\
\hline$\frac{1}{4}$ & $r^{4}-\frac{1037}{856} r^{2}$ & $\begin{array}{l}r_{1}^{2}=0.364366862 \\
r_{2}^{2}=0.847081739\end{array}$ & $\begin{array}{l}c_{1}=0.091808296 \\
c_{2}=0.157215143\end{array}$ \\
\hline & $+\frac{16909}{54784}$ & & \\
\hline$\frac{1}{2}$ & $r^{4}-\frac{29}{22} r^{2}$ & $\begin{array}{l}r_{1}^{2}=0.451910920 \\
r_{2}^{2}=0.866270899\end{array}$ & $\begin{array}{l}c_{1}=0.094048036 \\
c_{2}=0.140326967\end{array}$ \\
\hline & $+\frac{689}{1760}$ & & \\
\hline$\frac{3}{4}$ & $r^{4}-\frac{3956505}{2505272} r^{2}$ & $\begin{array}{l}r_{1}^{2}=0.664422219 \\
r_{2}^{2}=0.914849411\end{array}$ & $\begin{array}{l}c_{1}=0.077239032 \\
c_{2}=0.093659411\end{array}$ \\
\hline & $+\frac{487302501}{801687040}$ & & \\
\hline
\end{tabular}

$m=1, \quad n=5$

\begin{tabular}{l|l|l|l}
\hline 0 & $r^{4}-\frac{14}{11} r^{2}+\frac{35}{99}$ & $r_{1}^{2}=0.409596534$ & $c_{1}=0.065637839$ \\
$r_{2}^{2}=0.863130739$ & $c_{2}=0.134362161$ \\
$r^{4}-\frac{49 \times 227879}{8 \times 1092923} r^{2}$ & $r_{1}^{2}=0.413170409$ & $c_{1}=0.066037827$ \\
$r_{2}^{2}=0.863917619$ & $c_{2}=0.133766869$ \\
$+\frac{35 \times 31387427}{2816 \times 1092923}$ & & \\
$r^{4}-\frac{7 \times 53557}{22 \times 12601} r^{2}$ & $r_{1}^{2}=0.475583690$ & $c_{1}=0.070121263$ \\
$+\frac{35 \times 237793}{1584 \times 12601}$ & $r_{2}^{2}=0.876758471$ & $c_{2}=0.123628742$ \\
$r^{4}-\frac{7 \times 8334140936}{704 \times 52193401} r^{2}$ & $r_{1}^{2}=0.669472413$ & $c_{1}=0.065438853$ \\
$+\frac{35 \times 92932900132}{99 \times 1024 \times 52193401}$ & $r_{2}^{2}=0.918235562$ & $c_{2}=0.087100212$ \\
\hline
\end{tabular}


Let

$$
f\left(x_{1}, x_{2}, \cdots, x_{n}\right)=f_{1}\left(\theta_{1}, \theta_{2}, \cdots, \theta_{n-1}, r\right) .
$$

The Jacobian of the transformation is

$$
J\left(\theta_{1}, \theta_{2}, \cdots, \theta_{n-1}, r\right)=r^{n-1} \sin \theta_{2} \sin ^{2} \theta_{3} \cdots \sin ^{n-2} \theta_{n-1}
$$

$D$ is now defined by:

$$
\begin{aligned}
& 0 \leqq \theta_{1} \leqq 2 \pi ; 0 \leqq \theta_{i} \leqq \pi \quad(i=2,3, \cdots, n-1), \text { and } \\
& R \leqq r \leqq 1 .
\end{aligned}
$$

The rule to be constructed has the form:

$$
\int_{-}^{1} \int^{\pi} \cdots \int^{\pi} \int^{2 \pi} J f_{1}\left(\theta_{1}, \theta_{2}, \cdots, \theta_{n-1}, r\right) d \theta_{1} d \theta_{2} \cdots d \theta_{n-1} d r
$$

The first of these, (2.14), must be exact whenever $f_{1}$ is a polynomial of degree $k$ in

$$
\begin{aligned}
& r \sin \theta_{1} \sin \theta_{2} \cdots \sin \theta_{n-1}, \\
& r \cos \theta_{1} \sin \theta_{2} \cdots \sin \theta_{n-1}, \\
& \cdots \cdots \cdots \cdot \cdots \cdot \cdots \cdot \ln , \\
& r \cos \theta_{n-1}
\end{aligned}
$$

for $\theta_{2}, \theta_{3}, \cdots, \theta_{n-1}, r$ parameters; i.e., whenever $f_{1}$ is of degree $k$ in $\sin \theta_{1}$ and $\cos \theta_{1}$ and, hence, whenever $f_{1}=1, \cos \theta_{1}, \sin \theta_{1}, \cos 2 \theta_{1}, \sin 2 \theta_{1}, \cdots, \cos k \theta_{1}$ or $\sin k \theta_{1}$. This requirement leads to the set of $2 k+1$ equations:

$$
\left.\begin{array}{rl}
\sum_{g=1}^{p_{1}} a_{g} & =\int_{0}^{2 \pi} d \theta_{1}=2 \pi, \\
\sum_{g=1}^{p_{1}} a_{g} \cos t \theta_{1 g} & =\int_{0}^{2 \pi} \cos t \theta_{1} d \theta_{1}=0, \\
\sum_{g=1}^{p_{1}} a_{g} \sin t \theta_{1 g} & =\int_{0}^{2 \pi} \sin t \theta_{1} d \theta_{1}=0 .
\end{array}\right\} \quad(t=1,2, \cdots, k)
$$

One solution with minimum $p_{1}$ for given $k$ is

$$
\begin{aligned}
& p_{1}=k+1=4(m+1), \\
& a_{g}=\frac{2 \pi}{k+1}, \quad \theta_{1 g}=\frac{2 \pi g}{k+1}, \quad g=1,2, \cdots, k+1 .
\end{aligned}
$$

This is the only solution, apart from a single arbitrary additive constant to $\theta_{1 g}$, for which the $a_{g}$ are real so the above, $(2.18)$, is a necessary and sufficient condition for the first rule, (2.14), to have the required precision with a minimum number of points.

Put, temporarily,

$$
\begin{aligned}
\theta_{l}=\phi, \quad b_{l i}=b_{i}, \quad p_{l}=p, \quad l=\nu+1, & \text { and } \\
& f_{l}\left(\theta_{l}, \cdots, \theta_{n-1}, r\right)=g(\phi),
\end{aligned}
$$

so a typical member of the second set of rules, (2.15), is now 
NUMERICAL INTEGRATION OVER THE $n$-DIMENSIONAL SPHERICAL SHELL 583

$$
\alpha_{g h \cdots i j}=a_{g} b_{2, h} \cdots b_{n-1, i} c_{j},
$$

where

$$
\begin{aligned}
a_{g} & =\frac{2 \pi}{k+1}, \quad g=1,2, \cdots, k+1 \\
b_{l, i} & =\frac{\left(\left(2 m+1+\frac{1}{2} l\right) !\right)^{2}}{(2 m+2) !(2 m+l) !} \cdot \frac{2^{l-1}}{\left(1-y_{i}^{2}\right)\left(Q_{2 m+2}^{\prime l l 2}\left(y_{i}\right)\right)^{2}}, \\
\quad l=2, \cdots, n-1, \quad i=1,2, \cdots, 2 m+2, & \\
c_{j} & =\frac{1}{Q_{m+1}^{\prime}\left(r_{j}^{2}\right)} \int_{R}^{1} \frac{r^{n-1} Q_{m+1}\left(r^{2}\right) d r}{r^{2}-r_{j}^{2}} .
\end{aligned}
$$

Proof. Because the evaluation points of the rule (1.7) are arranged in a product lattice the integral can be written

$$
\begin{aligned}
I \equiv \int_{D} f(\mathbf{x}) d \tau=\int_{R}^{1} r^{n-1} \int_{0}^{\pi} & \sin ^{n-2} \theta_{n-1} \int_{0}^{\pi} \cdots \int_{0}^{\pi} \sin \theta_{2} \\
& \cdot \int_{0}^{2 \pi} f_{1}\left(\theta_{1}, \theta_{2}, \cdots, \theta_{n-1}, r\right) d \theta_{1} d \theta_{2} \cdots d \theta_{n-1} d r
\end{aligned}
$$

Define functions $f_{2}, \cdots, f_{n}$ as follows:

$$
\begin{aligned}
& f_{2}\left(\theta_{2}, \cdots, \theta_{n-1}, r\right)= \int_{0}^{2 \pi} f_{1}\left(\theta_{1}, \theta_{2}, \cdots, \theta_{n-1}, r\right) d \theta_{1}, \\
& f_{l}\left(\theta_{l}, \cdots, \theta_{n-1}, r\right)= \int_{0}^{\pi} \sin ^{l-2} \theta_{l-1} f_{l-1}\left(\theta_{l-1}, \cdots, \theta_{n-1}, r\right) d \theta_{l-1} \\
& \quad(l=3,4, \cdots, n-1), \\
& f_{n}(r)=\int_{0}^{\pi} \sin ^{n-2} \theta_{n-1} f_{n-1}\left(\theta_{n-1}, r\right) d \theta_{n-1} ;
\end{aligned}
$$

then

$$
I=\int_{R}^{1} r^{n-1} f_{n}(r) d r .
$$

We look for integration rules:

$$
\begin{aligned}
& \int_{0}^{2 \pi} f_{1} d \theta_{1} \doteq \sum_{g=1}^{p_{1}} a_{g} f_{1}\left(\theta_{1 g}, \theta_{2}, \cdots, \theta_{n-1}, r\right), \\
& \int_{0}^{\pi} \sin ^{l-1} \theta_{l} f_{l} d \theta_{l} \doteq \sum_{i=1}^{p_{l}} b_{l i} f_{l}\left(\theta_{l, i}, \theta_{l+1}, \cdots, \theta_{n-1}, r\right) \\
&(l=2, \cdots, n-1),
\end{aligned}
$$

and

$$
\int_{R}^{1} r^{n-1} f_{n}(r) d r \doteq \sum_{j=1}^{p_{n}} c_{j} f_{n}\left(r_{j}\right) .
$$


The first of these, $(2.14)$, must be exact whenever $f_{1}$ is a polynomial of degree $k$ in

$$
\begin{aligned}
& r \sin \theta_{1} \sin \theta_{2} \cdots \sin \theta_{n-1}, \\
& r \cos \theta_{1} \sin \theta_{2} \cdots \sin \theta_{n-1}, \\
& \cdots \cdots \cdots \cdots \cdots \cdots \\
& r \cos \theta_{n-1}
\end{aligned}
$$

for $\theta_{2}, \theta_{3}, \cdots, \theta_{n-1}, r$ parameters; i.e., whenever $f_{1}$ is of degree $k$ in $\sin \theta_{1}$ and $\cos \theta_{1}$ and, hence, whenever $f_{1}=1, \cos \theta_{1}, \sin \theta_{1}, \cos 2 \theta_{1}, \sin 2 \theta_{1}, \cdots, \cos k \theta_{1}$ or $\sin k \theta_{1}$. This requirement leads to the set of $2 k+1$ equations:

$$
\left.\begin{array}{rl}
\sum_{g=1}^{p_{1}} a_{g} & =\int_{0}^{2 \pi} d \theta_{1}=2 \pi, \\
\sum_{g=1}^{p_{1}} a_{g} \cos t \theta_{1 g} & =\int_{0}^{2 \pi} \cos t \theta_{1} d \theta_{1}=0, \\
\sum_{g=1}^{p_{1}} a_{g} \sin t \theta_{1 g} & =\int_{0}^{2 \pi} \sin t \theta_{1} d \theta_{1}=0 .
\end{array}\right\} \quad(t=1,2, \cdots, k)
$$

One solution with minimum $p_{1}$ for given $k$ is

$$
\begin{aligned}
& p_{1}=k+1=4(m+1), \\
& a_{g}=\frac{2 \pi}{k+1}, \quad \theta_{1 g}=\frac{2 \pi g}{k+1}, \quad g=1,2, \cdots, k+1 .
\end{aligned}
$$

This is the only solution, apart from a single arbitrary additive constant to $\theta_{1 g}$, for which the $a_{g}$ are real so the above, (2.18), is a necessary and sufficient condition for the first rule, (2.14), to have the required precision with a minimum number of points.

Put, temporarily,

$$
\begin{aligned}
\theta_{l}=\phi, \quad b_{l i}=b_{i}, \quad p_{l}=p, \quad l=\nu+1, & \text { and } \\
& f_{l}\left(\theta_{l}, \cdots, \theta_{n-1}, r\right)=g(\phi),
\end{aligned}
$$

so a typical member of the second set of rules, (2.15), is now

$$
\int_{0}^{\pi} \sin ^{\nu} \phi g(\phi) d \phi \doteq \sum_{i=1}^{p} b_{i} g\left(\phi_{i}\right), \quad \nu=1,2, \cdots, n-2,
$$

and is to be exact when the function $g(\phi)$ is a polynomial in $\sin \phi$ and $\cos \phi$ of degree at most $k$. The integral over the centrally symmetric $n$-spherical shell of any monomial $x_{1}{ }^{t_{1}} x_{2}{ }^{t_{2}} \cdots x_{n}{ }^{t_{n}}$ with an odd power $t_{s}$ is zero and is computed exactly by any rule centrally symmetric about the origin. So, provided rule (2.20) is symmetric, it needs to be exact only for all even polynomials in $\sin \phi$ and $\cos \phi$ of degree at most $k$ or, equivalently, for all polynomials in $\cos \phi$ alone of degree at most $k$. This requirement leads to the $k+1$ equations:

$$
\sum_{i=1}^{p} b_{i} \cos ^{t} \phi_{i}=\int_{0}^{\pi} \sin ^{\nu} \phi \cos ^{t} \phi d \phi, \quad t=0,1, \cdots, k .
$$


Change the variable to $y$ by the equations

$$
y=\cos \phi \quad \text { and } \quad y_{j}=\cos \phi_{j},
$$

and (2.21) becomes

$$
\sum_{i=1}^{p} b_{i} y_{i}^{t}=\int_{-1}^{1}\left(1-y^{2}\right)^{(\nu-1) / 2} y^{t} d y, \quad t=0,1, \cdots, k .
$$

These are the equations determining a rule

$$
\int_{-1}^{1}\left(1-y^{2}\right)^{(\nu-1) / 2} h(y) d y \doteq \sum_{i=1}^{p} b_{i} h\left(y_{i}\right)
$$

of precision $k$ in $y$ and with weight function $\left(1-y^{2}\right)^{(\nu-1) / 2}$. Now the unique rule of precision $2 p-1$ using a minimum number of points for the weight function $(1-y)^{\alpha}(1+y)^{\beta}$ over the interval $(-1,1)$ is the $p$-point Gauss-Mehler rule, which has abscissae at the zeros of the orthogonal polynomials

$$
Q_{p}{ }^{(\alpha, \beta)}(y) \equiv\left(\begin{array}{c}
p+\alpha \\
p
\end{array}\right) G_{p}\left(\alpha+\beta+1, \alpha+1, \frac{1}{2}(1-y)\right)
$$

where $G_{p}(a, b, z)$ are the Jacobi polynomials defined by

$$
G_{p}(a, b, z) \equiv F(-p, p+a ; b, z),
$$

where $F(a, b ; c, z)$ is the ordinary hypergeometric series (terminating in this case) and which has weights [2]

$$
\begin{aligned}
& b_{i}=\frac{1}{{Q_{p}}^{(\alpha, \beta)}\left(y_{i}\right)} \int_{-1}^{1} \frac{Q_{p}^{(\alpha, \beta)}(y) d y}{y-y_{i}} \\
& =\frac{(p+\alpha) !(p+\beta) !}{p !(p+\alpha+\beta) !} \cdot \frac{2^{\alpha+\beta+1}}{\left(1-y_{i}{ }^{2}\right)\left(Q^{\prime(\alpha, \beta)}\left(y_{i}\right)\right)^{2}} \cdot
\end{aligned}
$$

For the rule (2.24)

$$
\alpha=\beta=(\nu-1) / 2 \quad(\nu=1,2, \cdots, n-2),
$$

and for brevity write

$$
Q_{p}^{(\alpha, \alpha)}(y)=Q_{p}^{\alpha}(y) .
$$

It follows that for the rule (2.24) to be of precision $4 m+3$ with a minimum number of points, $p$, that $p=2 m+2$, the abscissae $y_{i}$ are the $2 m+2$ zeros of

$$
Q_{2 m+2}^{(\nu-1) / 2}(y)=\left(\begin{array}{c}
2 m+2+(\nu-1) / 2 \\
2 m+2
\end{array}\right) G_{2 m+2}\left(\nu, \frac{\nu+1}{2}, \frac{1-y}{2}\right),
$$

and the weights are

$$
b_{i}=\frac{\left(\left(2 m+2+\frac{\nu-1}{2}\right) !\right)^{2}}{(2 m+2) !(2 m+\nu+1) !} \cdot \frac{2^{\nu}}{\left(1-y_{i}^{2}\right)\left(Q_{2 m+2}^{\prime(\nu-1) / 2}\left(y_{i}\right)\right)^{2}} .
$$

On replacing $\nu$ by $l-1, y$ by $\cos \theta$, etc., according to (2.19) one gets the result in the second part of the theorem. 
Because of the symmetry of the angular integration rules all the monomials with an odd power of some coordinate $x_{i}$ have their integrals (zero) computed exactly, independently of rule (2.16), so rule (2.16) need be exact only for all even polynomials in $r$ of degree at most $4 m+3$, i.e., one must have

$$
\int_{R}^{1} r^{n-1} g\left(r^{2}\right) d r \doteq \sum_{j=1}^{q} c_{j} g\left(r_{j}^{2}\right)
$$

exact whenever $g\left(r^{2}\right)$ is a polynomial in $r^{2}$ of degree at most $2 m+1$ and with $q$ a minimum. Put $r^{2}=t$ and this becomes

$$
\int_{R^{2}}^{1} t^{n / 2-1} g(t) d t \doteq \sum_{j=1}^{q} 2 c_{j} g\left(t_{j}\right)
$$

The rule $(2.32)$ of precision $2 m+1$ and minimum number of points, $q$, is unique [3] and has $q=m+1$. The $t_{j}$ are the $m+1$ zeros of the polynomial $Q_{m+1}(t)$ in $t$ of degree $m+1$ that is orthogonal with respect to the weight function $t^{n / 2-1}$ over $\left(R^{2}, 1\right)$ to all polynomials in $t$ of lower degree; i.e.,

$$
\int_{R^{2}}^{1} t^{n / 2-1} Q_{m+1}(t) T_{m}(t) d t=0,
$$

where $T_{m}(t)$ is an arbitrary polynomial of degree at most $m$, and the weights $2 c_{j}$ are given by:

$$
2 c_{j}=\frac{1}{Q_{m+1}^{\prime}\left(t_{j}\right)} \int_{R^{2}}^{1} \frac{t^{n / 2-1} Q_{m+1}(t) d t}{t-t_{j}} .
$$

On replacing $t$ by $r^{2},(2.33)$ and (2.34) become (2.4) and (2.8), respectively, in the last part of the statement of the theorem. This completes the proof.

\section{Discussion.}

CoROLlary 1. The weights $\beta_{g h \cdots i}=a_{g} b_{2, h} \cdots b_{n-1, i}$ are positive and their sum is

$$
\frac{2 \pi^{n / 2}}{\left(\frac{n}{2}-1\right) !}
$$

the area of the surface of the unit $n$-sphere.

CoRollary 2. The weights $\alpha_{g h \cdots i j}=a_{g} b_{2, h} \cdots b_{n-1, i} c_{j}$ are positive and their sum is

$$
\frac{1-R^{n}}{n} \cdot \frac{2 \pi^{n / 2}}{\left(\frac{n}{2}-1\right) !}
$$

the volume of the n-spherical shell.

Corollary 3. All evaluation points of the rule lie between the inner and outer $n$-spherical surfaces of the shell.

CoRollary 4. Integration rule (2.14) uses $4(m+1)$ points; the $n-2$ rules (2.15) each use $2(m+1)$ points and rule $(2.16)$ uses $m+1$ points so the number of points used by the complete rule (1.7) is

$$
(4(m+1))(2(m+1))^{n-2}(m+1) \text { or } 2^{n}(m+1)^{n} .
$$


Peirce's result of $8(m+1)^{3}$ points for the three-dimensional shell is recovered by taking $n=3$.

Corollary 5. When, in the integration rule $(2.24), \nu$ is odd:

$$
\nu=2 l+1 \quad l=0,1,2, \cdots,
$$

then in (2.25) $\alpha=\beta=l$, an integer, and the rule becomes a Gauss-Christoffel rule for which the $Q_{p}{ }^{l}(y)$ can be written in terms of the Legendre polynomials $P_{n}(y)$ :

$$
\left(1-y^{2}\right)^{l} Q_{p}{ }^{l}(y)=\left|\begin{array}{cccc}
P_{p}(y) & P_{p+2}(y) & \cdots & P_{p+2 l}(y) \\
P_{p}(1) & P_{p+2}(1) & \ldots & P_{p+2 l}(1) \\
P_{p}^{\prime}(1) & P_{p+2}^{\prime}(1) & \ldots & P_{p+2 l}^{\prime}(1) \\
\ldots \ldots \ldots & \ldots \ldots \ldots \ldots & \ldots & \ldots \\
P_{p}{ }^{(l-1)}(1) & P_{p+2}^{(l-1)}(1) & \cdots & P_{p+2 l}^{(l-1)}(1)
\end{array}\right|
$$

where [2], [3]

$$
P_{n}^{(l)}(y) \equiv \frac{d^{l}}{d y^{l}} P_{n}(y)
$$

When $\nu=1$ the rule is the classical Gauss' one with $p=2 m+2, y_{i}=\cos \phi_{i}$, the zeros of the Legendre polynomial $P_{2 m+2}(y)$ and

$$
b_{i}=\frac{2}{\left(1-y_{i}^{2}\right)\left(P_{2 m+2}^{\prime}\left(y_{i}\right)\right)^{2}} .
$$

For the integral over the three-dimensional shell $\nu$ takes just the single value 1. This particular case is the one given by Peirce [1].

When $\nu=3$ one gets from (3.1) and the properties of $P_{n}(y)$

$$
Q_{p}{ }^{1}(y)=\frac{2 p+3}{(p+1)(p+2)} \cdot P_{p+1}^{\prime}(y)
$$

so the zeros of $Q_{p}{ }^{1}(y)$ are the same as the zeros of $P_{p+1}^{\prime}(y)$.

Corollary 6. When $\nu$ is even put

$$
\nu=2 \lambda, \quad \lambda=1,2, \cdots,
$$

then

$$
\alpha=\beta=\lambda-\frac{1}{2}
$$

and

$$
Q_{p}{ }^{\alpha}(y)=Q_{p}^{\lambda-1 / 2}(y)=\left(\begin{array}{c}
p+\lambda-\frac{1}{2} \\
p
\end{array}\right) G_{p}\left(2 \lambda, \lambda+\frac{1}{2}, \frac{1}{2}(1+y)\right) .
$$

For the case $\lambda=1$ the polynomial is a particularly simple one and has the explicit form for all $p$ [2]:

$$
Q_{p}^{1 / 2}(\cos \phi)=2 \frac{1 \cdot 3 \cdot 5 \cdots(2 p+1)}{2 \cdot 4 \cdot 6 \cdots(2 p+2)} \cdot \frac{\sin (p+1) \phi}{\sin \phi}
$$

which has zeros:

$$
\phi_{i}=\frac{\pi i}{p+1}
$$


The associated weights are

$$
b_{i}=\frac{\pi\left(1-\cos ^{2} \phi_{i}\right)}{p+1} .
$$

The values for $\phi_{i}$ have the great practical advantage, as have those of the $\theta_{1 g}$, of being equally spaced.

The ordinary hypergeometric function $F(a, b ; c, z)$ satisfies the relation

$$
\frac{d}{d z} F(a, b ; c, z)=\frac{a b}{c} F(a+1, b+1 ; c+1, z),
$$

so from the definition (2.26) of the Jacobi polynomials, $G_{p}(a, b, z)$,

$$
\frac{d}{d z} G_{p+1}(a, b, z)=\frac{-(p+1)(p+1+a)}{b} G_{p}(a+2, b+1, z) .
$$

From (3.10):

$$
G_{p}\left(2\left(\alpha+\frac{3}{2}\right), \alpha+2, z\right)
$$

$$
=\frac{-(\alpha+1)}{(p+1)(p+2(\alpha+1))} \cdot \frac{d}{d z} G_{p+1}\left(2\left(\alpha+\frac{1}{2}\right), \alpha+1, z\right) .
$$

Now, using (2.25), (2.28) and (3.11), one finds

$$
Q_{p}^{\alpha+1}(y)=\left(\begin{array}{c}
p+\alpha+1 \\
p
\end{array}\right) \frac{-(\alpha+1)}{(p+1)(p+2(\alpha+1))} \cdot \frac{d}{d z} G_{p+1}\left(2\left(\alpha+\frac{1}{2}\right), \alpha+1, z\right)
$$

where $z=(1-y) / 2$ so

$$
Q_{p}^{\alpha+1}(y)=\frac{-1}{(p+2(\alpha+1))} \cdot \frac{d}{d z} Q_{p+1}^{\alpha}(y) .
$$

From this

$$
Q_{p}^{\alpha+1}(y)=\frac{2}{p+2(\alpha+1)} \cdot \frac{d}{d y} Q_{p+1}^{\alpha}(y),
$$

a useful differential recursion relation.

One can use this relation to find $Q_{p}^{3 / 2}$ from $Q_{p+1}^{1 / 2}$ :

$$
Q_{p}^{3 / 2}=\frac{2}{p+3} \frac{d}{d y} Q_{p+1}^{1 / 2}(y)
$$

where $y=\cos \phi$ so, by (3.6),

$$
Q_{p}{ }^{3 / 2}=\frac{2}{p+3} \cdot 2 \cdot \frac{1 \cdot 3 \cdot 5 \cdots(2 p+3)}{2 \cdot 4 \cdot 6 \cdots(2 p+4)} \cdot \frac{d}{d \phi}\left(\frac{\sin (p+2) \phi}{\sin \phi}\right) \cdot \frac{d \phi}{d y} .
$$

Now

$$
\begin{aligned}
& \frac{d}{d \phi}\left(\frac{\sin (p+2) \phi}{\sin \phi}\right) \cdot \frac{d \phi}{d y} \\
& =\frac{2}{2 \sin ^{3} \phi}((p+3) \sin (p+1) \phi-(p+1) \sin (p+3) \phi)
\end{aligned}
$$


so the zeros of $Q_{p}^{3 / 2}$ are the roots (in $(0, \pi)$ ) of

$$
(p+3) \sin (p+1) \phi-(p+1) \sin (p+3) \phi=0 .
$$

This does not have a simple explicit solution for all the $\phi_{i}$ and for all the relevant $p$ so it is unlikely that any $Q_{p}^{\lambda-1 / 2}$ where $\lambda>1$ has such a solution. For each value of $p$ a separate solution has to be found. A few are given in Table I.

Corollary 7. When $R=0$ the radial rule (2.32) becomes

$$
\int_{0}^{1} t^{n / 2-1} g(t) d t \doteq \sum_{j=1}^{m+1} 2 c_{j} g\left(t_{j}\right)
$$

and the polynomials $Q_{m+1}(t)$ of equations (2.4) and (2.8) are simply the Jacobi polynomials $G_{m+1}(n / 2, n / 2, t)$.

For $n=3$ Peirce [1] has tabulated the $Q_{m+1}(t), t_{j}$ and $c_{j}$ for $m=0$ and 1 (also for $R=\frac{1}{4}, \frac{1}{2}$ and $\frac{3}{4}$ ). For $n$ an even number, $n / 2-1$ is an integer and (3.17) is a Gauss-Christoffel rule for which the $Q_{m+1}(t)$ can be obtained either from the Legendre polynomials (but with argument $2 t-1$ ) as in (3.1) or as a Jacobi polynomial above. The tables of Fishman [4] give normalised $Q_{m+1}(t)$ and values of $t_{j}$ and $2 c_{j}$ above for $n=2,4,6, \cdots, 12$ and for $m=0,1,2, \cdots, 7$. (His $n$ is my $n / 2-1$ and his $m$ is my $m+1$.)

Table II contains some values of $r_{j}^{2}$ and $c_{j}$ for $m=0,1$. (Note: for $n$ an odd number Fishman's tables could be used directly - treating the sphere as a cone with a spherical base - to give an integration rule but the number of points used in such a rule would not be a minimum since it would unnecessarily compute exactly integrals of certain odd powers of $r$.)

Corollary 8. If the integration rule (1.2) is modified to include the weight function $r^{s}$ ( $s$ an integer), all the foregoing is still applicable except that in (2.4), (2.8), etc., $n+s$ must be substituted for $n$ so, for example, the table of $c_{j}$ and $r_{j}$ for the five-dimensional shell can be used for the three-dimensional shell with weight function $r^{2}$.

The author is grateful to the referee for drawing his attention to the work in References 5 and 6.

School of Mathematics

University of New South Wales

Sydney, New South Wales,

Australia

1. W. H. Peirce, "Numerical integration over the spherical shell," MTAC, v. 11, 1957, p. 244-249.

2. Z. Kopal, Numerical Analysis, Wiley, New York, 1955, p. 367-386.

3. G. SzEgö, Orthogonal Polynomials, Amer. Math. Soc. Colloq. Publ. Vol. 23, Amer. Math. Soc., Providence, R. I., 1939.

4. H. FishMAN, "Numerical integration constants," MTAC, v. 11, 1957, p. 1-9.

5. A. H. STroud \& D. SeCrest, "Approximate integration formulas for certain spherically symmetric regions," Math. Comp., v. 17, 1963, p. 105-135.

6. I. P. Mrsovskin, "Cubature formulas for evaluating integrals over a sphere," Dokl. Akad. Nauk SSSR, v. 147, No. 3, 1962, p. 552-555. (Russian) 